\title{
Schizencephaly - A case report
}

\author{
P. K. Chhetri ${ }^{1}$, S. Raut ${ }^{2}$
}

${ }^{1}$ Assistant Professor, ${ }^{2}$ Junior Resident, Department of Radiodiagnosis, College of Medical Sciences, Bharatpur, Chitwan district, Nepal

\begin{abstract}
Schizencephaly is an uncommon congenital cerebral malformation that involves the cerebral mantle and consists of a cleft that extends through the entire cerebral hemisphere from the lateral ventricle to cerebral cortex. The condition is present at birth and present early in life. Here we present an adult patient with schizencephaly presenting with first onset seizure.
\end{abstract}

Key words: schizencephaly, lateral ventricle, seizure imaging diagnosis

\section{Introduction}

Schizencephaly (split brain) is a gray matter-lined cerebrospinal fluid - filled cleft that extends from the ependymal surface of the brain through the white matter to the pia. There is no well-documented data on its incidence. ${ }^{1}$ Two types are recognized, which have prognostic significance. In type I or closed-lip schizencephaly, the cleft walls are in apposition and type II or open lip schizencephaly, in which the walls are separated. Schizencephaly type II occurs more commonly than type I. ${ }^{1}$

In either instance the cleft is lined by heterotopic gray matter. In most cases, the gray matter along the cleft is polymicrogyric; in some instances, it is more dysplastic than polymicrogyric. ${ }^{2}$ The clefts can be unilateral or bilateral, symmetric or asymmetric and can appear anywhere in the brain, although they usually are perisylvian. In unilateral cases, perusal of the contralateral hemisphere is warranted, as subtle clefts of polymicrogyria are common. ${ }^{2}$

Correspondence: Dr. P K Chhetri

E-mail:pramodchhetri@rediffmail.com

\section{Case Report}

A 50 year old male patient was referred for CT scan of the head for a single episode of a generalized tonic - clonic seizure. Apart from mental retardation present since birth no other significant history could be elicited. A non - contrast enhanced CT scan of the head was performed. Axial (Figure 1) and coronal (Figure 2) CT scan shows the wide open - lip schizencephaly with communication of the left lateral ventricle and the subarachnoid space on the left side. There is also absent septum pellucidum and partial thinning of the skull vault on the left side (temporoparietal region).

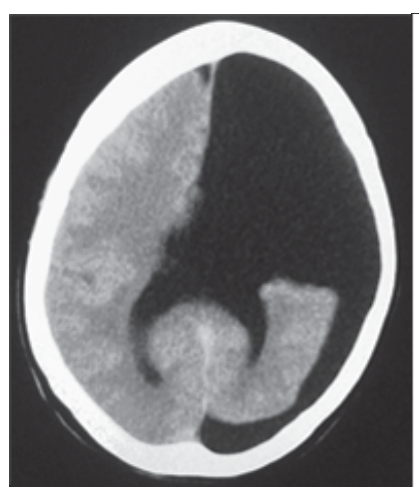

Figure 1

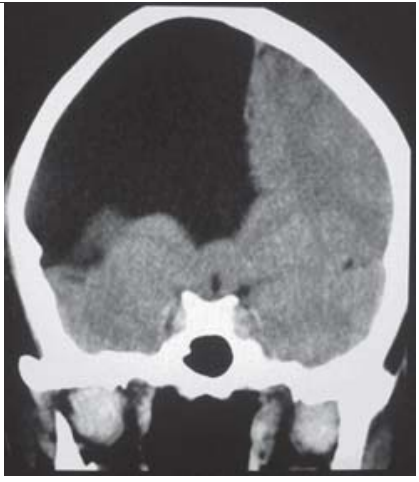

Figure 2 
P. K. Chhetri et al. Schizencephaly - A case report

\section{Discussion}

Several theories have been proposed to explain the etiology of schizencephaly, although none is universally accepted. However, an ischemic episode occurring at the $7^{\text {th }}$ week of gestation has been hypothesized as an etiological factor. In the normal embryo, beginning $7^{\text {th }}$ week of gestation, neuroblasts are generated in the germinal matrix. During the $8^{\text {th }}$ week these primitive cells begin to migrate along radially oriented glial cells to the cerebral cortical regions. During that critical period, any insult to the centripetal and centrifugal vessels in the region of germinal matrix may cause hypoxaemia and infarction with arrest of migration of these neuroblasts. ${ }^{3}$ Conversely, another author favours a primary developmental abnormality to account for the combination of schizencephaly and absence of the cavum septum pellucidum. ${ }^{4}$ Recently, mutations in the homebox gene, Emx2 located on chromosome 10q2.6, have been reported in some patients with schizencephaly. ${ }^{2}$

Presenting symptoms relate to the amount of involved brain. Children with closed-lip schizencephaly typically present with hemiparesis or motor delay, whereas patients with open-lip schizencephaly usually present with hydrocephalus or seizures. Patient with closed-lip schizencephaly are more likely to have mild to moderate neurologic deficit than those with openlip type. Unilateral closed-lip schizencephaly is associated with the best neurodevelopmental outcome. Patients with progressively larger clefts present with progressively greater neurologic deficits because progressively more brain is missing. Nearly all patients with bilateral open-lip clefts have severe disabilities. ${ }^{2}$

CT scans of closed-lip schizencephaly may show only a slight outpouching, or "nipple,"at the ependymal surface of the cleft. The full thickness cleft, or pialependymal seam, may be difficult to detect on CT scans but is easily discernible on MR studies. Openlip schizencephaly has a larger, more apparent gray matter-lined CSF cleft. ${ }^{5}$

CT may show subependymal or parenchymal calcification in many cases, which suggests that one cause of schizencephaly may be intrauterine infection with cytomegalovirus. ${ }^{6}$

MRI is the imaging modality of choice because of its superior differentiation of gray matter and white matter and its ability to image in more than one plane. Identification of gray matter lining the cleft is the pathognomic finding. ${ }^{1}$

MRI shows the abnormal appearance of the cortical mantle along the cleft and the cortex appearing thicker than normal owing to the presence of polymicrogyria. The contralateral hemisphere may also have developmental abnormalities, such as polymicrogyria and subependymal heterotopias. ${ }^{6}$

Mild hypoplasia of the corpus callosum is commonly seen. The septum pellucidum is absent or nearly completely absent in $70 \%$ to $90 \%$ of affected patients. Of those with absence of the septum pellucidum, 30-50\% will have optic nerve hypoplasia on clinical examination. Therefore, septo-optic dysplasia is, by definition, present in $20-45 \%$ of patients with schizencephaly. ${ }^{2}$ Optic atrophy is usually easily recognizable clinically but is often difficult to confirm on imaging.

In addition to the brain anomalies, in patients with openlip schizencephaly, CSF pulsations from the lateral ventricles result in pressure effects on the inner table of the skull. ${ }^{2}$ 


\section{References}

1. Bird CR, Gilles FH. Type 1 schizencephaly: CT and neuropathologic findings. Am J of Neuroradio 1987; Vol $8,(3): 451-4$

2. Robertson R, Caruso PA, Truwit CL et al Disorders of Brain Development. In: Magnetic Resonance Imaging

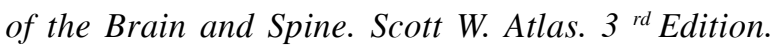
Lippincott Williams \& Wilkins, 2002;356-60

3. Chuang SH, Fitz CR, Chilton SJ et al: Schizencephaly; Spectrum of CT findings in association with septo-optic dysplasia. Presented at the 70 th Scientific assembly and annual meeting of Radiological Society of North
America Washington DC November. Radiology 1984;153:118

4. Page LK, Brown SB, Gargano FP et al. Schizencephaly: a clinical study for review. Child Brain 1975;1:348-58

5. Anne G. Osborn. Disorders of Diverticulation and Cleavage, Sulcation and Cellular Migraton. In: Diagnostic Neuroradiology. Mosby, 1994;52-4

6. Gunny RS, Chong WKK. Paediatric Neuroradiology. In: Grainger \& Allison's Diagnostic Radiology. 5 th Edition. Volume II. Churchill Livingstone Elsevier, 2008; 1660 